Heloisa Giangrossi Machado Vidotti ${ }^{\text {a }}$

Marina Greghi Sticca ${ }^{b}$

Talita Naiara Rossi da Silva ${ }^{\mathrm{c}}$

Nilton Luiz Menegon ${ }^{\mathrm{a}}$

a Universidade Federal de São Carlos, Departamento de Engenharia de Produção. São Carlos, SP, Brasil.

b Universidade de São Paulo, Departamento de Psicologia. Ribeirão Preto, SP, Brasil.

' Universidade Federal de Minas Gerais, Departamento de Terapia Ocupacional. Belo Horizonte, MG, Brasil.

Contato:

Heloisa Giangrossi Machado Vidotti

E-mail:

helogm@yahoo.com.br

O trabalho não é baseado em tese ou dissertação e não foi apresentado em reunião científica.

Os autores declaram que o trabalho não foi subvencionado e que não há conflitos de interesses.

\section{Trabalho e saúde dos comissários de bordo: uma revisão}

\author{
Work and health of flight attendants: a review
}

\section{Resumo}

Introdução: com o aumento do número de voos e passageiros, as tarefas atribuídas aos comissários de bordo tornam-se mais complexas, podendo gerar sobrecarga e prejuízo à saúde e ao desempenho desses trabalhadores. Objetivos: identificar e sistematizar os artigos publicados em periódicos científicos, sobre o impacto de fatores físicos, ambientais e organizacionais do trabalho dos comissários de bordo na saúde desses trabalhadores. Método: revisão sistemática da literatura, em português e inglês, utilizando as palavras-chave "aeronave", "cabine", "transporte aéreo", "comissário de bordo" e "trabalho", nas bases de dados ScienceDirect, PubMed, Scopus, ProQuest e SciELO, considerando o período de 1983 a 2013. Resultados: 65 artigos atenderam aos critérios de inclusão. Verificou-se um predomínio de trabalhos com foco em fatores físicos e/ou relacionados à saúde/doença e em fatores relacionados à satisfação no trabalho e poucos estudos abordando a organização do trabalho. Conclusão: os estudos encontrados analisaram os fatores presentes nas situações de trabalho de comissários de bordo de forma isolada. Não foram encontrados estudos que adotassem uma metodologia de análise das situações de trabalho que possibilitasse estabelecer inter-relações entre os fatores determinantes da carga de trabalho desses trabalhadores e os impactos para sua saúde.

Palavras-chave: comissário de bordo; trabalho; saúde; revisão; aviação comercial.

\begin{abstract}
Introduction: with the increase in the number of flights and passengers, the tasks carried out by flight attendants become more complex, and may cause overload and damage to their health and performance. Objective: to identify articles and systematize information published in scientific journals related to flight attendants' health impact of the physical, environmental and organizational factors of their work. Method: systematic review of the scientific literature, in Portuguese and English, using the keywords "aircraft", "cabin", "air transportation", "flight attendant" and "work" in ScienceDirect, PubMed, Scopus, ProQuest and SciELO databases, published between 1983-2013. Results: 65 articles met the inclusion criteria. There was a predominance of studies focusing on physical factors and/or factors related to health/disease and related to job satisfaction, with few studies addressing work organization. Conclusion: the studies investigated the relationship between the work environment and the health of flight attendants using limited approaches. No studies were found adopting an analysis methodology that had allowed stablishing relationships between determining factors of workload and their impacts on the flight attendants health.
\end{abstract}

Keywords: flight attendant; work; health; review; commercial aviation. 


\section{Introdução}

A demanda por transporte aéreo vem crescendo no Brasil ${ }^{1}$. No ano de 2012, o mercado de voos domésticos atingiu o maior nível nos últimos dez anos, com aproximadamente 989 mil voos realizados e cerca de 88 milhões de passageiros transportados, representando aumentos de $85 \%$ e $205 \%$, respectivamente, comparados a 2003. Nesse setor, cerca de 19\% do total de empregados são comissários de bordo, o que corresponde a aproximadamente 12 mil profissionais atuando em companhias aéreas brasileiras ${ }^{1}$.

Considerando o crescimento do setor, observa-se um aumento da demanda por transporte aéreo e, como possível consequência, aumento das horas de voo e serviços da tripulação. No Brasil, o regime de trabalho do aeronauta é regulado pela Lei $\mathrm{n}^{\circ} 7.183$, de 5 de abril de $1984^{2}$, regulamentada pela Portaria Interministerial $\mathrm{n}^{\circ} 3.016$, de 5 de fevereiro de $1988^{3}$, expedida pelo Ministério do Trabalho e Ministério da Aeronáutica. Essa lei dispõe sobre a escala de serviço, jornada de trabalho, horas de voo, limites do número de pousos por dia para cada comissário e períodos de repouso.

Apesar das regulamentações nacionais e internacionais, a literatura tem apontado diversos fatores presentes no ambiente de trabalho que afetam a saúde dos comissários de bordo. Nessa perspectiva, um estudo ${ }^{4}$ analisou dados do Centro de Medicina Aeroespacial (Cemal), do Ministério da Aeronáutica do Brasil, e verificou que, no ano de 2001, 2,19\% dos comissários do sexo masculino e 1,76\% do sexo feminino estavam incapacitados definitivamente para a função, enquanto $15,41 \%$ dos comissários do sexo feminino e $8,52 \%$ do sexo masculino estavam incapacitados temporariamente.

Outro estudo ${ }^{5}$ mostrou que entre os anos de 2002 e 2007, no Hospital da Aeronáutica de São Paulo, foram realizadas 648 perícias em comissários de bordo relacionadas a diagnóstico de transtorno mental ou comportamental associado ou não a distúrbio ou transtorno orgânico, entre as quais 336 geraram afastamentos. Além disso, no estudo observa-se que cerca de um terço dos afastamentos são causados por transtornos mentais, indicando que a carga psíquica é um importante fator para o adoecimento desses profissionais. Em outra pesquisa ${ }^{6}$, foram realizadas entrevistas com nove comissários de bordo, e foram relatados diversos prejuízos relacionados à saúde, como dor no estômago, dor nas pernas, inchaço, varizes, dor nas costas e bursite, entre outros.

Portanto, sistematizar os achados sobre o tema faz-se importante para planejar pesquisas futuras que possam integrar os fatores físicos e organizacionais relacionados à satisfação e à situação de trabalho, identificando, assim, os aspectos causadores de sobrecarga para esses trabalhadores.

Desse modo, este estudo tem como pergunta principal "Como a literatura científica tem tratado a questão dos fatores relativos ao trabalho que impactam a saúde de comissários de bordo?", tendo como objetivo identificar e sistematizar artigos, publicados em periódicos científicos, sobre o impacto de fatores físicos, ambientais e organizacionais do trabalho dos comissários de bordo na saúde desses trabalhadores.

\section{Método}

Realizou-se revisão sistemática da literatura sobre o trabalho de comissários de bordo a partir da análise de artigos científicos disponíveis nas bases eletrônicas de dados ScienceDirect, PubMed, Scopus, ProQuest e SciELO, escolhidas por serem referência nas áreas de saúde e engenharia, disciplinas que abordam o tema. Foram utilizadas as palavras-chave "comissário de bordo" (flight attendant) combinada com "aeronave" (aircraft), "cabine" (cabin), "transporte aéreo" (air transportation) e "trabalho" (work). A pesquisa bibliográfica foi realizada no período compreendido entre abril de 2014 e abril de 2015, considerando trabalhos publicados de 1983 a 2013.

Para a organização e sistematização dos artigos encontrados foi utilizado o software StArt ${ }^{7}$, versão 1.6.3, desenvolvido pelo Laboratório de pesquisa em Engenharia de Software da Universidade Federal de São Carlos (UFSCar). Essa ferramenta auxilia o planejamento, execução e análise final dos artigos encontrados, tornando a revisão sistemática mais ágil, precisa e replicável ${ }^{8}$. Para sua utilização, é necessário o preenchimento de um protocolo com as possíveis características dos estudos, assim como critério de inclusão/exclusão.

Esse protocolo constitui um dos diferenciais entre as revisões tradicionais e as sistemáticas, pois enquanto as primeiras se limitam às conveniências do pesquisador na seleção da literatura para o seu estudo - que não necessariamente seguem critérios definidos - as segundas se valem de uma pergunta clara, a definição de uma estratégia de busca, o estabelecimento de critérios de inclusão e exclusão dos artigos e uma análise criteriosa da qualidade da literatura selecionada ${ }^{9}$.

Ao encontrar pesquisas de interesse, os dados são importados para o software diretamente da base eletrônica de dados, no formato Bibtex. Dessa forma, o software tem acesso aos autores, resumos e anos de publicação dos estudos, que são classificados conforme as características inseridas no protocolo. Assim, como relatório final, o software classifica os artigos a ser analisados e com maior enquadramento nos critérios de inclusão. 
Após a estratégia de busca ser realizada, os artigos encontrados foram avaliados independentemente por dois autores, seguindo os critérios de inclusão: a) população-alvo (comissários de bordo); b) exigências físicas do trabalho; c) características ambientais do trabalho; d) características organizacionais do trabalho dos comissários de bordo; e) efeitos do trabalho para a saúde desses trabalhadores; f) estudos escritos em inglês e português. Os critérios de exclusão foram: a) estudos que, apesar de apresentarem como tema o transporte aéreo, não abordavam o trabalho do comissário de bordo; b) capítulos de livros, teses e dissertações.

Para a seleção inicial dos artigos foi realizada uma análise com base nos títulos dos manuscritos. Nos casos em que não foi possível identificar os critérios de inclusão pelo título, os resumos foram analisados e, em seguida, tomava-se a decisão de incluir ou excluir o artigo neste trabalho. Após análise dos resumos, os estudos foram obtidos na íntegra e, posteriormente, examinados de acordo com os critérios de inclusão estabelecidos. Os processos de seleção e avaliação foram realizados por pares de pesquisadores.

As categorias de análises dos dados foram elaboradas e validadas por três avaliadores, escolhidos por serem pesquisadores da área de ergonomia e saúde do trabalhador, com atuação na área de aviação. A partir da leitura dos artigos, sugeriram categorias temáticas e, por meio de consenso, em três reuniões de discussão, categorizaram os artigos selecionados. Foram elaboradas duas categorias temáticas predominantes: "Fatores relativos à situação de trabalho" e "Doenças relacionadas ao trabalho", divididas conforme os subtemas identificados nos estudos selecionados.

\section{Resultados e discussão}

Combinando-se todos os métodos de busca, foram identificados 858 estudos, dos quais 65 preencheram os critérios de inclusão e foram selecionados.
Todos tinham origem europeia e estadunidense, com predomínio de estudos cujo objetivo primordial era a descrição das características do ambiente de trabalho dos comissários de bordo, sem o estabelecimento de relações entre variáveis e nem da natureza dessas relações.

Os trabalhos selecionados foram sistematizados segundo as seguintes informações: base bibliográfica, autor e período de publicação; tipo e metodologia de estudo; população de estudo, principais resultados e frequência de resultados.

A Tabela 1 mostra o número de artigos encontrados e selecionados em cada base de dados e o período em que foram publicados.

A Tabela 2 descreve os estudos selecionados em relação ao tipo de delineamento de pesquisa ${ }^{10}$. Em relação aos delineamentos da produção científica, 16 estudos foram baseados em dados secundários, com predominância de revisão da literatura e pesquisa documental. Constatou-se predomínio da pesquisa de levantamento (19 estudos), que tem como característica a abordagem metodológica quantitativa. Também foram observados diversos trabalhos com abordagem qualitativa, como estudos de casos (14 estudos). De modo geral, considerando as características de todos os artigos, houve predominância da abordagem qualitativa (36 estudos).

Na Tabela 3 são sistematizados os temas levantados nas produções científicas das cinco bases eletrônicas de dados analisadas. Verificou-se um predomínio de artigos com foco em fatores físicos e/ou relacionados à saúde/doença ( 25 estudos) e em fatores relacionados à satisfação no trabalho de comissários de bordo (21 estudos), sendo que outros 13 trabalhos abordam os dois temas simultaneamente. O tema "organização do trabalho" representa a minoria dos artigos (6 trabalhos).

Tabela 1 Quantidade de artigos sobre saúde e trabalho de comissários de bordo encontrados e selecionados, segundo as bases de dados pesquisadas e o período de publicação

\begin{tabular}{|c|c|c|c|c|c|c|}
\hline \multirow[b]{2}{*}{ Base de dados } & \multirow{2}{*}{$\begin{array}{c}\text { Número de artigos } \\
\text { encontrados }\end{array}$} & \multirow[b]{2}{*}{ Número de artigos selecionados } & \multicolumn{4}{|c|}{ Período da publicação } \\
\hline & & & 1983-1989 & 1990-1999 & 2000-2009 & 2010-2013 \\
\hline ScienceDirect & 568 & 25 & 2 & 0 & 14 & 9 \\
\hline Scopus & 153 & 31 & 1 & 3 & 24 & 3 \\
\hline PubMed & 40 & 7 & 0 & 1 & 3 & 3 \\
\hline ProQuest & 94 & 2 & 0 & 1 & 0 & 1 \\
\hline SciELO & 03 & 0 & 0 & 0 & 0 & 0 \\
\hline TOTAL & 858 & 65 & 3 & 5 & 41 & 16 \\
\hline
\end{tabular}


Tabela 2 Metodologia e tipo de pesquisa dos artigos selecionados sobre saúde e trabalho de comissários de bordo, publicados entre 1983 e 2013

\begin{tabular}{|c|c|c|}
\hline Critério de classificação & $\begin{array}{c}\text { Número de } \\
\text { artigos }\end{array}$ & Artigos encontrados \\
\hline \multicolumn{3}{|l|}{ Metodologia* } \\
\hline Levantamento (survey) & 19 & $\begin{array}{l}\text { Haugli e cols. }{ }^{11} \text {; Lindgren e cols. }{ }^{12} \text {; Sonnentag e Natter }{ }^{13} \text {; Ballard e cols. }{ }^{14} \text {; Chen }{ }^{15} \text {; Heuven e } \\
{\text { cols. }{ }^{16} \text {; Lee e cols }{ }^{17} \text {; Chang e Liao }}^{18} \text {; Lee e cols. }{ }^{19} \text {; Shao e cols. }{ }^{20} \text {; Chung e Chung }{ }^{21} \text {; Yeh }{ }^{22} \text {; Chen e } \\
\text { Kao }^{23} \text {; Ng e cols. }{ }^{24} \text {; Wahlstedt e cols. }{ }^{25} \text {; Chen e Kao }{ }^{26} \text {; Chen e Kao }{ }^{27} \text {; Lee e cols. } .^{28} \text {; Moon e cols. }{ }^{29}\end{array}$ \\
\hline Estudo de caso & 14 & 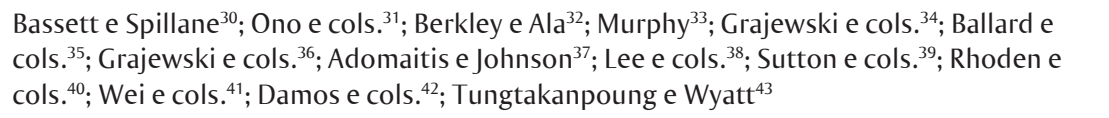 \\
\hline Revisão da literatura & 10 & $\begin{array}{l}\text { Boyd e Bain }{ }^{44} \text {; Ballard e cols. }{ }^{45} \text {; Hocking }{ }^{46} \text {; Nagda e Koontz }{ }^{47} \text {; Megdal e cols. }{ }^{48} \text {; Buja e cols. }{ }^{49} \text {; } \\
\text { Leggat e Smith }{ }^{50} \text {; Tokumaru e cols. } .^{51} \text {; Michels }{ }^{52} \text {; Griffiths e Powell }{ }^{53}\end{array}$ \\
\hline Pesquisa documental & 6 & Iglesias e cols. ${ }^{54}$; Gill ${ }^{55}$; Linnersjö e cols..$^{56}$; Waters e cols..$^{57}$; Banks e cols. ${ }^{58}$; Pinkerton e cols. ${ }^{59}$ \\
\hline Outros & 16 & 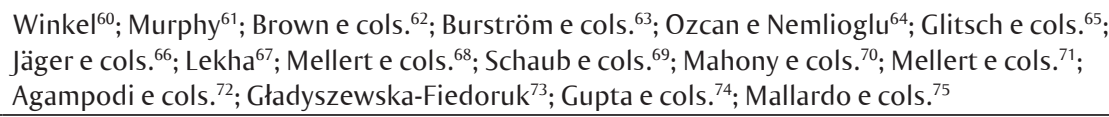 \\
\hline \multicolumn{3}{|l|}{ Tipo de Pesquisa } \\
\hline Qualitativa & 36 & 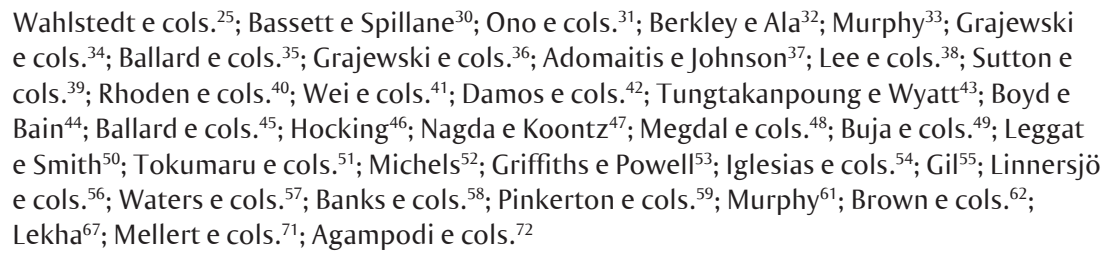 \\
\hline Quantitativa & 29 & 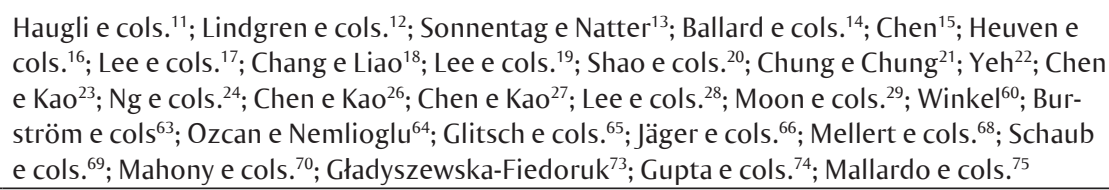 \\
\hline
\end{tabular}

${ }^{*}$ Segundo Gil ${ }^{10}$.

Tabela 3 Temas abordados nos artigos selecionados sobre saúde e trabalho de comissários de bordo, publicados entre 1983 e 2013

\begin{tabular}{|c|c|c|}
\hline Tema & Número de artigos & Artigos encontrados \\
\hline Organização do trabalho & 6 & $\begin{array}{l}\text { Berkley e Ala }{ }^{32} \text {; Grajewski e cols. }{ }^{36} \text {; Rhoden e cols. }{ }^{40} \text {; Griffiths e Pow- } \\
\text { ell }^{53} \text {; Gil }{ }^{55} \text {; Mahony e cols. }{ }^{70}\end{array}$ \\
\hline $\begin{array}{l}\text { Fatores físicos e/ou } \\
\text { relacionados à saúde/ } \\
\text { doenças }\end{array}$ & 25 & 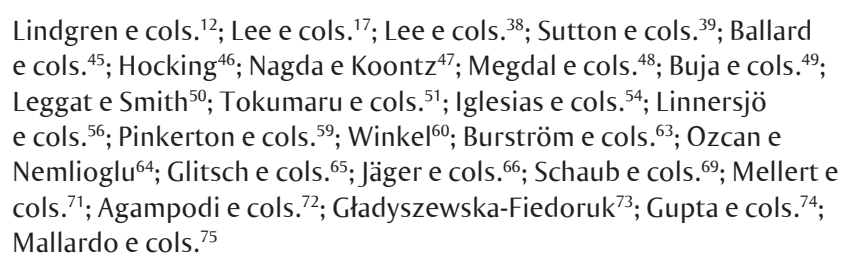 \\
\hline $\begin{array}{l}\text { Fatores relacionados à } \\
\text { satisfação no trabalho }\end{array}$ & 21 & 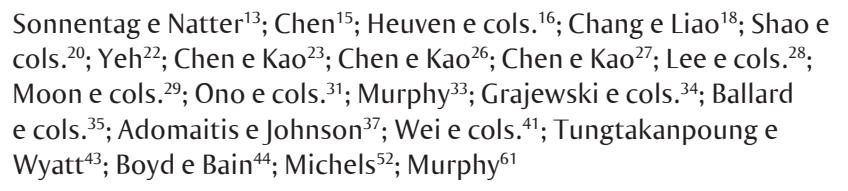 \\
\hline $\begin{array}{l}\text { Fatores físicos relacio- } \\
\text { nados à satisfação no } \\
\text { trabalho }\end{array}$ & 13 & $\begin{array}{l}\text { Haugli e cols. }{ }^{11} \text {; Ballard e cols. }{ }^{14} \text {; Lee e cols. }{ }^{19} \text {; Chung e Chung }{ }^{21} \text {; Ng e } \\
\text { cols. }{ }^{24} \text {; Wahlstedt e cols. } .^{25} \text {; Bassett e Spillane }{ }^{30} \text {; Damos e cols. }{ }^{42} \text {; Wa- } \\
\text { ters e cols. }{ }^{57} \text {; Banks e cols. }{ }^{58} \text {; Brown e cols. }{ }^{62} \text {; } \text { Lekha }^{67} \text {; Mellert e cols. }{ }^{68}\end{array}$ \\
\hline
\end{tabular}


Com relação às categorias e subcategorias temáticas definidas pelos analistas especialistas, os trabalhos foram classificados e discutidos como apresentado a seguir.

\section{Fatores relativos à situação de trabalho}

Os estudos encontrados apontam fatores relativos à situação de trabalho que podem influenciar a saúde e o desempenho do comissário de bordo. Esses fatores podem ser categorizados em:

- aspectos relacionados ao ambiente físico da cabine $^{47,63,64,71,72}$;

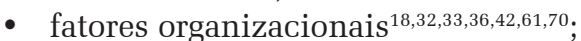

- fatores biomecânicos ${ }^{17,38,60,65,66,69}$;

- fatores relacionados à satisfação no trabalho ${ }^{15,22,24,26,27,43}$;

\section{Fatores relacionados ao ambiente físico da cabine}

Estudos apontam as influências do ambiente físico da cabine na saúde física dos comissários de bordo $^{38}$. Entre os fatores destacados pelos autores estão a vibraçãa ${ }^{63,71}$, os ruídos na cabine ${ }^{64,71,75}$ e os fatores relacionados ao desconforto muscular e ao conforto da cabine ${ }^{47,72}$.

Em dois trabalhos verificou-se que a vibração transmitida aos comissários durante o voo estava relacionada a lesões e desconforto no pescoço, ombro e coluna lombar ${ }^{63,71}$. Os resultados apontaram que os efeitos adversos são potencializados pelos múltiplos pousos aos quais os comissários estão submetidos. Além disso, constatou-se que o maior nível de vibração ocorre na parte traseira da aeronave, logo, os trabalhadores que se localizavam nessa posição estavam mais propensos a seus efeitos.

Além da vibração, a análise do nível de ruído também foi objeto de outros estudos ${ }^{64,68,71,75}$, segundo os quais o nível de ruído interfere na saúde física e psicológica, pois, quando há excesso de ruído durante a jornada de trabalho, sintomas como dor de cabeça, irritação, ansiedade, distração e dores musculares são relatados pelos comissários de bordo. Verificou-se que os valores mais elevados do nível de ruído acontecem no pouso e na decolagem, sendo constantes - por volta de $78 \mathrm{~dB}$ - durante o voo, a não ser quando o comandante realiza algum comunicado. Nas situações em que o nível de ruído aumenta, foi observado o aumento proporcional dos sintomas relatados pelos comissários de bordo, como dor de cabeça e irritação.

Em uma revisão ${ }^{47}$, identificaram-se estudos que analisaram o conforto dos comissários de bordo na cabine durante os voos, destacando-se sintomas provocados pelo ambiente da cabine, como secura relacionada à baixa umidade do ar, inchaço e dor de cabeça/ouvido causados pelo ambiente hipobárico.

Também foram encontrados estudos que tinham como objeto identificar fatores presentes nas cabines da aeronave que podem influenciar diretamente no trabalho e na saúde dos comissários de bordo. Dos cinco artigos encontrados em relação a esse tema $^{12,46,62,73,74}$, quatro deles abordam a questão da qualidade do ar no interior da cabine.

Em outro estudo ${ }^{73}$ foi encontrado durante um voo excesso de $\mathrm{CO}_{2}$ (comparado ao padrão da Organização Mundial da Saúde) no ar após três horas de viagem, o que pode provocar fadiga, dor de cabeça, problemas de concentração e outros desconfortos. Além disso, a temperatura estava acima do nível de conforto, podendo ocasionar problemas de concentração para a tripulação. Essas características do ar acontecem principalmente porque os sistemas de ventilação das aeronaves priorizam a regulação da pressão e, por consequência, a qualidade do ar permanece em segundo plano, sendo um dos fatores mais preocupantes em relação à saúde dos comissários de bordo ${ }^{62}$.

Um artigo ${ }^{46}$ destaca que, para minimizar os custos com combustível, os voos são realizados em altitudes cada vez mais elevadas. Com isso, é necessário aumentar a recirculação de ar, o que pode diminuir sua qualidade. Desse modo, para que essa qualidade não seja comprometida, sugere-se a substituição dos filtros simples por filtros de alta eficiência de partículas de ar (HEPA), mais eficazes, o que diminuiria a proliferação de agentes infecciosos.

Esse mesmo assunto é abordado em outro estudo $^{74}$, no qual se identificou a possibilidade de contaminação do ambiente da cabine por partículas de RNA do vírus Influenza. Assim, a solução sugerida seria o uso de máscaras pela tripulação e passageiros.

\section{Fatores organizacionais}

Além dos fatores físicos e dos relacionados à satisfação no trabalho, os aspectos organizacionais do trabalho podem afetar negativamente a saúde dos comissários de bordo. Poucos estudos abordam a sobrecarga proporcionada pelo volume de tarefas atribuídas a eles, relativas à segurança dos passageiros, do próprio voo e ao serviço de bordo.

Para garantir a segurança dos passageiros, os comissários devem realizar procedimentos específicos. Por exemplo, devem checar se os passageiros estão utilizando os cintos de segurança durante turbulências, decolagem e aterrissagem e devem zelar pela limpeza da cabine para evitar que objetos voem durante uma turbulência. Caso ocorra alguma lesão 
ou mal-estar entre os passageiros, os comissários devem proceder conforme o treinamento específico para essas situações ${ }^{18,70}$.

As tarefas relacionadas à segurança do voo têm como objetivo identificar e neutralizar atividades que coloquem em risco o andamento normal do voo $^{32,40}$. Por fim, os comissários de bordo também realizam o serviço de bordo e atendem a eventuais pedidos dos passageiros ${ }^{42}$.

Depois do 11 de setembro de 2001, os procedimentos de segurança do voo e dos passageiros se tornaram mais complexos e rigorosos ${ }^{42}$. Dessa forma, a maior parte do tempo do voo deve ser dedicada aos procedimentos de segurança. Porém, por causa das crises econômicas e flutuações de demanda, os comissários de bordo são obrigados a dispenderem mais tempo no serviço aos passageiros para torná-los clientes regulares da companhia aérea. Essa mudança de perspectiva favorece a sobrecarga de trabalho, uma vez que a tripulação é reduzida e não consegue realizar todas as tarefas, além de colocar a segurança do voo em segundo plano ${ }^{42}$.

Também no contexto da organização do trabalho, torna-se importante ressaltar estudos que mostraram as diferenças existentes entre as horas trabalhadas relatadas pelos comissários de bordo e as horas registradas pelas companhias aéreas. $\mathrm{O}$ relato preciso das horas de trabalho pode ser utilizado como parâmetro para medir a exposição dos trabalhadores a variáveis como radiação cósmica e ciclo circadiano ${ }^{36,58}$.

\section{Fatores Biomecânicos}

Estudos ${ }^{60,65,66,69}$ analisaram a sobrecarga biomecânica muscular ao empurrar/puxar os carrinhos de serviço. Recentemente, o serviço de bordo foi estendido para a fase ascendente e descendente do voo, o que ocasiona maior sobrecarga muscular ao conduzir os carrinhos de serviço ${ }^{69}$. Foram identificadas queixas relatadas pelos comissários de bordo em relação a dores musculares, principalmente na coluna lombar ${ }^{66}$. Também foram analisadas as forças necessárias para a condução do carrinho de serviço, comparadas ao limite máximo de força que pode ser exercida sem prejuízo à saúde. Os estudos encontrados concluíram que as forças exercidas no serviço de bordo ao conduzir o carrinho ficaram acima dos limites recomendados, principalmente quando estão cheios ou em uma superfície íngreme ${ }^{69}$.

Os carrinhos menores causam menos danos à saúde física do que os maiores ${ }^{60,66,69}$. Já com relação às disfunções musculares, dependem da inclinação da superfície, do tipo de carrinho, de seu peso, se a força é exercida para empurrar ou puxar e da frequência das operações ${ }^{69}$. Assim, quanto maior a inclinação da superfície, peso do carrinho e o movimento de puxar, maior a sobrecarga nos músculos e na coluna vertebral ${ }^{65,69}$.

No uso do carrinho durante o serviço de bordo, o comissário realiza frequentes flexões de coluna para servir o passageiro, aumentando significativamente a sobrecarga muscular ${ }^{17}$. Um grupo de trabalhadores relatou que posturas instáveis, como agachamento, alcance e ficar em pé por longos períodos, aumentam as desordens musculares relacionadas ao trabalho.

No entanto, salienta-se que o manuseio do carrinho de serviço não é o único fator físico que interfere na saúde dos comissários de bordo, mas que problemas musculares também podem ser ocasionados por outras tarefas exercidas nessa profissão. Por exemplo, o manuseio de bagagens dos passageiros é considerado uma das tarefas mais extenuantes, pois é realizada frequentemente e requer esforços para empurrar, puxar e levantar, provocando sobrecarga muscular ${ }^{17}$.

Outro artigo analisou os principais causadores de acidentes de trabalho de comissários de bordo ${ }^{72}$, no qual foram identificados os riscos de quedas, escorregamento e tropeço, além de outras lesões durante o voo. Os principais fatores associados às lesões foram: turbulência, serviço com o carrinho, auxílio com a bagagem, bagageiros e outros. No estudo foram identificadas 795 lesões em cada 1.000 comissários de bordo por ano, o que foi considerada alta prevalência. Essa mesma evidência foi encontrada em outro estudo $^{54}$, que identificou tais lesões como responsáveis por 15.573 dias de trabalho perdidos no período de 4 anos (entre 1983 e 1987), em uma amostra de 1.631 comissários de bordo.

\section{Fatores relacionados à satisfação no trabalho}

Os principais fatores que levam os comissários de bordo a deixarem o trabalho são o salário, estado civil, insatisfação com o trabalho, compromisso no cumprimento das normas e necessidade de dedicação contínua ${ }^{15,26}$.

Desse modo, níveis altos de satisfação com o trabalho e compromisso organizacional apresentam impacto negativo na intenção de abandonar o emprego, ou seja, quanto maior a satisfação com o trabalho e maior o compromisso organizacional, menor a probabilidade de o comissário de bordo desistir do emprego ${ }^{15}$.

A satisfação com o trabalho consiste em fatores relacionados à motivação, característica da atividade, autoridade e responsabilidade; ao ambiente (condições, supervisão e colegas); às características organizacionais (salário, estabilidade, promoção e política organizacional); e à dimensão social (prestígio, reputação e responsabilidade social) ${ }^{28}$. Os 
trabalhadores que apresentam níveis altos de satisfação com o trabalho demonstram afetividade e maior possibilidade de contribuir com a organização, sendo que esses esforços levarão a um melhor desempenho na função.

O compromisso do trabalhador com o trabalho depende de fatores como crenças e identificação com o trabalho. Aqueles que demonstram compromisso apresentam vontade de permanecer no ramo. Os comissários de bordo com altos níveis de compromisso e orgulho da profissão podem aumentar a qualidade de serviços prestados, assim como a imagem da companhia aérea perante os passageiros. Dessa forma, para incentivar o maior compromisso da parte dos trabalhadores, é necessário um adequado clima organizacional, o que contribui para o desempenho no trabalho e aumento da qualidade de serviço percebido pelos passageiros ${ }^{22,24,43}$.

\section{Doenças relacionadas ao trabalho}

Doenças ocasionadas por estresse psicológico podem ser relacionadas ao trabalho dos comissários de bordo ${ }^{25,35}$. Outros exemplos podem ser encontrados, como problemas respiratórios e de fertilidade ${ }^{53}$, desequilíbrio do ciclo circadiano ${ }^{34-57}$, problemas digestivos, dermatite ${ }^{50}$, disfunção circulatória em membros inferiores, infecções no trato urinário, alguns tipos de câncer ${ }^{45,48,49,51,53,56,59}$, e intoxicação por pesticidas utilizados na desinfecção da cabine ${ }^{39,41}$.

Em outro trabalho ${ }^{67}$ foram encontrados sintomas como estresse, dores nas costas, perda de memória, dores de cabeça e perda de audição em comissários de bordo, enquanto em outro estudo ${ }^{11}$ foram encontrados problemas como secura da pele, dor na coluna lombar, resfriados, fadiga e distúrbio do sono.

Além disso, foram observados altos níveis de cortisol - hormônio que se apresenta em altas quantidades em situações estressantes - na urina dos comissários de bordo, tanto antes como depois da jornada de trabalho ${ }^{30}$. Antes do trabalho, esse hormônio estava alterado possivelmente pela ansiedade pré-voo, enquanto depois da jornada o principal motivo poderia estar relacionado à disfunção do ciclo circadiano.

Também foram encontrados estudos que apontaram sobrecarga psicológica, relatada em um número relevante de doenças, como estresse e síndrome do esgotamento profissional ${ }^{23,26}$. Um deles ${ }^{23}$ apontou as relações entre os fatores que causam o estresse no trabalho e suas possíveis consequências, entre os quais os conflitos familiares causados pela longa jornada de trabalho, que afetam direta ou indiretamente a satisfação com o trabalho, o compromisso organizacional e a intenção de deixar o emprego.
Da mesma forma, outro estudo ${ }^{26}$ analisou empiricamente a relação entre demanda de trabalho, recursos, síndrome do esgotamento profissional, isolamento dos colegas, problemas de saúde e desempenho no trabalho. Observou-se que as demandas de trabalho são as principais causas da síndrome de esgotamento profissional, o que, por sua vez, pode afetar a saúde do trabalhador. Adicionalmente, as pressões incompatíveis entre trabalho e família produzem tensões que provocam exaustão. Assim, interferências familiares e a falta de estabilidade no emprego são responsáveis diretas pelo aparecimento da síndrome de esgotamento profissional e, consequentemente, diminuição do desempenho no trabalho.

Além disso, um estudo ${ }^{27}$ investigou como o engajamento no trabalho e a estabilidade no emprego podem influenciar o desenvolvimento da síndrome de esgotamento profissional. Foi identificado que quanto maior o compromisso com o trabalho, maior a probabilidade do aparecimento da síndrome, o que diminui o desempenho no trabalho. Além disso, os comissários de bordo com maior estabilidade no emprego resistem melhor a essa síndrome.

Segundo alguns autores ${ }^{35}$, sintomas como depressão, ansiedade e ataque de pânico foram relatados por comissárias de bordo. Ainda segundo esses relatos, frequentemente há o surgimento de estresse na readaptação da família após longos períodos no trabalho. Uma comissária participante do estudo relatou situações de divórcio, pois acredita que os cônjuges não oferecem apoio suficiente para conciliar o trabalho com a criação dos filhos e a realização de tarefas domésticas. Além disso, as comissárias de bordo que têm filhos relataram a sensação de culpa, pois não conseguem frequentar eventos importantes para a formação deles. Dessa forma, segundo os autores, são identificados o estresse e sofrimento mental relacionados à solidão, medo de serem parceiros e pais inadequados por causa da demanda de trabalho, dificuldade na relação com o passageiro e falta de estabilidade no emprego ${ }^{35}$.

Alguns estudos ${ }^{14,16,21,29}$ discutem a sobrecarga emocional relacionada à profissão do comissário de bordo, na qual podem ocorrer assédio sexual e moral por parte dos superiores e passageiros, enfrentamento com passageiros agressivos, respostas às emergências ocorridas durante o voo e, principalmente para as comissárias que já tiveram filhos, medo de voar.

Esses trabalhadores são treinados para atuar, demonstrar aos passageiros sentimentos que não são verdadeiros, ou seja, suprimir os reais sentimentos. A maioria relata separar a vida pessoal do papel realizado durante o trabalho, porém quando essa 
separação deixa de ser claramente delineada, distúrbios mentais como exaustão emocional podem surgir. Para evitar tais situações, estratégias como não se envolver emocionalmente com o trabalho ${ }^{29}$ e controlar as emoções relativas à profissão ${ }^{16}$ demonstraram ser eficazes para evitar a exaustão emocional.

Dores nas costas de origem psicológica foram identificadas como resultado do estresse enfrentado pelos trabalhadores ${ }^{19}$. Os comissários que relataram dores nas costas apresentavam maior percepção das demandas psicológicas do trabalho, insegurança no emprego e sobrecarga física. O estresse ocasionado pela insegurança no trabalho, após descartada a sobrecarga física, é o fator mais importante no aparecimento das dores nas costas.

\section{Considerações finais}

A partir da análise dos estudos encontrados sobre o trabalho de comissários de bordo, verificou-se a predominância de artigos publicados entre 2000 e 2010, o que mostra uma preocupação recente com as condições de trabalho desses profissionais.

Constatou-se que condições ambientais presentes no interior da aeronave podem gerar sobrecarga física e psíquica. Também foram encontrados estudos que relacionavam aspectos organizacionais com sobrecarga de trabalho. Os estudos sobre doenças relacionadas ao trabalho de comissários de bordo apontaram doenças físicas, mas também doenças mentais.

Verificou-se ainda uma predominância de estudos do tipo levantamento, mais especificamente, survey. Não foram encontrados estudos que utilizassem como abordagem metodológica a análise ergonômica do trabalho, que é realizada por meio de uma investigação detalhada da atividade real dos trabalhadores, dentro do contexto em que ocorre, visando relacionar todos os fatores que influenciam na saúde do trabalhador e nas atividades de trabalho ${ }^{76}$.

A partir dos estudos encontrados, que analisaram o papel das companhias aéreas na organização do trabalho e, consequentemente, no aparecimento de doenças relacionadas, pode-se concluir que o trabalho de comissários de bordo parece ter impactos negativos sobre sua saúde e qualidade de vida, ocasionando doenças e sofrimento. No entanto, os fatores que podem ocasionar esses problemas foram predominantemente estudados de forma isolada, sem buscar identificar as inter-relações entre eles. Assim, parece existir uma lacuna de estudos sobre as inter-relações entre esses fatores, que, caso fossem realizados, permitiriam investigar o processo saúde-doença desses profissionais de forma mais completa.

Além disso, não foram encontrados estudos nacionais sobre a relação entre saúde e trabalho dos comissários de bordo. Os estudos encontrados foram desenvolvidos em outros países. Essa investigação seria importante para que fosse possível comparar a prevalência e os fatores associados ao desenvolvimento de doenças, considerando as demandas e características do transporte aéreo brasileiro. Cada país difere no que diz respeito à legislação e aos aspectos trabalhistas da área e, portanto, as atividades de comissários de bordo podem variar de maneira significativa entre um país e outro, ou mesmo entre companhias aéreas de um mesmo país.

Dessa forma, a partir da análise da literatura da área, verificou-se a necessidade da realização de estudos que identifiquem as inter-relações entre os fatores presentes na situação de trabalho de comissários de bordo e os processos de saúde-adoecimento, sobretudo no Brasil. A partir do estudo dessas inter-relações espera-se que se torne possível sugerir mudanças e melhorias das condições de trabalho desses profissionais.

\section{Contribuições de autoria}

Todos os autores contribuíram igualmente para o desenvolvimento do trabalho, incluindo o delineamento do projeto, levantamento de dados, análise e interpretação, elaboração e revisão do manuscrito.

\section{Referências}

1. Ministério da Aeronáutica, Agência Nacional de Aviação Civil (ANAC) (Brasil). Anuário Estatístico do Transporte Aéreo. Volume Único, 2012. Disponível em http:/www2.anac.gov.br/estatistica/ anuarios.asp.
2. Brasil. Lei $n^{0} 7.183$, de 5 de abril de 1984. Regula o exercício da Profissão de Aeronauta, e dá outras providências. Diário Oficial [da] República Federativa do Brasil, Poder Executivo, Brasília; 6 abr 1984. Seção 1, p. 4.969. 
3. Brasil. Portaria Interministerial $n^{\circ} 3.016$, de 5 de fevereiro de 1988. Expede instruções para execução da Lei 7.183, de 5 de abril de 1984, que dispõe sobre o exercício da profissão de aeronauta. Diário Oficial [da] República Federativa do Brasil, Brasília; 5 fev 1988.

4. Palma A. Ciência pós-normal, saúde e riscos dos aeronautas: a incorporação da vulnerabilidade. Tese [Doutorado em Ciências na aérea de Saúde Pública] - Fundação Oswaldo Cruz, Rio de Janeiro; 2002.

5. Nery MLC. Frequência e prevalência de diagnósticos psiquiátricos determinantes do afastamento de comissários de bordo da atividade aérea. Dissertação [Mestrado em Saúde Pública] Faculdade de Saúde Pública, Universidade de São Paulo, São Paulo; 2009.

6. Pimentel VL. Ser comissário de bordo: os significados destes profissionais acerca das adversidades do trabalho. Dissertação [Mestrado em Enfermagem] - Escola de Enfermagem da Universidade de São Paulo; 2006.

7. Fabbri S, Ferrari F, Vincenzi AMR, Di Thommazo A. State of the Art through Systematic Review (StArt). Versão 1.6.3 [software]. Laboratório de pesquisa em Engenharia de Software da Universidade Federal de São Carlos. 2013 [citado em 2015 fev 19]. Disponível em http://lapes. dc.ufscar.br/tools/start_tool

8. Zamboni AB, Di Thommazo A, Hernandes EC, Fabbri S. StArt: uma Ferramenta Computacional de Apoio à Revisão Sistemática. In: Anais do Congresso Brasileiro de Software: Teoria e Prática - Sessão de ferramentas; 2010 Set 27 a Out 1; Salvador: Universidade Federal da Bahia, 2010.

9. Sampaio RF, Mancini, MC. Estudos de revisão sistemática: um guia para síntese criteriosa da evidência científica. Rev bras fisioter. 2007;11(1):83-9.

10. Gil AC. Como elaborar projetos de pesquisa. 4. ed. São Paulo: Atlas; 2002.

11. Haugli L, Skogstad A, Hellesoy OH. Health, sleep, and mood perceptions reported by airline crews flying short and long hauls. Aviat Space Environ Med. 1994;65(1):27-34.

12. Lindgren T, Norbäck D, Andersson K, Dammström BG. Cabin environment and perception of cabin air quality among commercial aircrew. Aviat Space Environ Med. 2000;71(8):774-82.

13. Sonnentag S, Natter E. Flight attendants' daily recovery from work: is there no place like home? IJSM. 2004;11(4):366-91.

14. Ballard TJ, Romito P, Lauria L, Vigiliano V, Caldora M, Mazzanti C, et al. Self perceived health and mental health among women flight attendants. Occupational and Environmental Medicine. 2006;63(1):33-8.

15. Chen CF. Job satisfaction, organizational commitment, and flight attendants' turnover intentions: a note. JATM. 2006;12(5):274-6.
16. Heuven E, Bakker AB, Schaufeli WB, Huisman $\mathrm{N}$. The role of self-efficacy in performing emotion work. J Vocat Behav. 2006;69(2):222-35.

17. Lee H, Wilbur J, Conrad KM, Mokadam D. Workrelated musculoskeletal symptoms reported by female flight attendants on long-haul flights. Aviat Space Environ Med. 2006;77(12):1283-7.

18. Chang YH, Liao MY. Air passenger perceptions on exit row seating and flight safety education. Safety Science. 2008;46(10):1459-68.

19. Lee H, Wilbur J, Kim MJ, Miller AM. Psychosocial risk factors for work-related musculoskeletal disorders of the lower-back among long-haul international female flight attendants. J Adv Nurs. 2008;61(5):492-502.

20. Shao PC, Yen JJ, Ye KD. Identifying fatigue of flight attendants in short-haul operations. In: Proceedings of the 26th Congress of International Council of the Aeronautical Sciences including the 8th AIAA Aviation Technology, Integration, and Operations (ATIO) Conference; 2008 Sep 14-19; Anchorage, Alaska, USA. 2008. Paper ICAS 20089.2.3.

21. Chung CT, Chung UL. An exploration of quality of life and related factors among female flight attendants. JNR. 2009;17(3):212-20.

22. Yeh CW. Service climate, professional commitment and job performance of flight attendants in Taiwan. JATM. 2009;15(5):259-60.

23. Chen CF, Kao YL. The antecedents and consequences of job stress of flight attendants: evidence from Taiwan. JATM. 2011;17(4):253-5.

24. Ng SI, Sambasivan M, Zubaidah S. Antecedents and outcomes of flight attendants' job satisfaction. JATM. 2011;17(5):309-13.

25. Wahlstedt K, Lindgren T, Norbäck D, Wieslander G, Runeson R. Psychosocial work environment and medical symptoms among swedish commercial airline cabin crew. Am J Ind Med. 2010;53(7):71623.

26. Chen CF, Kao YL. Investigating the antecedents and consequences of burnout and isolation among flight attendants. Tourism Management. 2012;33(4):868-74.

27. Chen CF, Kao YL. Moderating effects of work engagement and job tenure on burnout: performance among flight attendants. JATM. 2012;25:61-3.

28. Lee C, An M, Noh Y. The social dimension of service workers' job satisfaction: the perspective of flight attendants. JSSM. 2012;5(2):160.

29. Moon TW, Hur WM, Jun JK. The role of perceived organizational support on emotional labor in the airline industry. International Journal of Contemporary Hospitality Management. 2013;25(1):105-23.

30. Bassett JR, Spillane R. Urinary cortisol excretion and mood ratings in aircraft cabin crew during a tour of duty involving a disruption in 
circadian rhythm. Pharmacol Biochem Behav. 1987;27(3):413-20.

31. Ono, Y, Watanabe S, Kaneko S, Matsumoto K, Miyao M. Working hours and fatigue of Japanese flight attendants (FA). J Hum Ergol. 1991;20:15564.

32. Berkley BJ, Ala M. Identifying and controlling threatening airline passengers. The Cornell Hotel and Restaurant Administration Quarterly. 2001;42(4):6-23.

33. Murphy AG. The flight attendant dilemma: An analysis of communication and sensemaking during in-flight emergencies. Journal of Applied Communication Research. 2001;29(1):30-53.

34. Grajewski B, Nguyen MM, Whelan EA, Cole RJ, Hein MJ. Measuring and identifying large-study metrics for circadian rhythm disruption in female flight attendants. Scand J Work Environ Health. 2003;29(5):337-46.

35. Ballard TJ, Corradi L, Lauria L, Mazzanti C, Scaravelli G, Sgorbissa F, et al. Integrating qualitative methods into occupational health research: a study of women flight attendants. Occup Environ Med. 2004;61(2):163-6.

36. Grajewski B, Atkins DJ, Whelan EA. Self-reported flight hours vs. company records for epidemiologic studies of flight attendants. Aviat Space Environ Med. 2004;75(9):806-10.

37. Adomaitis AD, Johnson KKP. Casual versus formal uniforms: flight attendants' self-perceptions and perceived appraisals by others. CTRJ. 2005;23:88101.

38. Lee H, Wilbur J, Conrad KM, Miller AM. Risk factors associated with work-related musculoskeletal disorders among female flight attendants: using a focus group to prepare a survey. AAOHN J. 2006;54(4):154-64.

39. Sutton PM, Vergara X, Beckman J, Nicas M, Das R. Pesticide illness among flight attendants due to aircraft disinsection. Am J Ind Med. 2007;50(5):345-56.

40. Rhoden S, Ralston R, Ineson EM. Cabin crew training to control disruptive airline passenger behavior: a cause for tourism concern? Tourism Management. 2008;29(3):538-47.

41. Wei B, Mohan KR, Weisel CP. Exposure of flight attendants to pyrethroid insecticides on commercial flights: urinary metabolite levels and implications. Int J Hyg Environ Health. 2012;215(4):465-73.

42. Damos DL, Boyett KS, Gibbs P. Safety versus passenger service: the flight attendants' dilemma. Int J Aviat Psychol. 2013;23(2):91-112.

43. Tungtakanpoung M, Wyatt M. Spirituality and cultural values in the reported cognitions of female cabin attendants on Thai Airways. JATM. 2013;27:15-9.

44. Boyd C, Bain P. Once I get you up there, where the air is rarified: health, safety and the working conditions of airline cabin crews. New Technology Work and Employment. 1998;13(1):16-28.

45. Ballard T, Lagorio S, De Angelis G, Verdeccia A. Cancer incidence and mortality among flight personnel: a meta-analysis. Aviat Space Environ Med. 2000;71(3):216-24.

46. Hocking MB. Passenger aircraft cabin air quality: trends, effects, societal costs, proposals. Chemosphere. 2000;41(4):603-15.

47. Nagda NL, Koontz MD. Review of studies on flight attendant health and comfort in airliner cabins. Aviat Space Environ Med. 2003;74(2):101-9.

48. Megdal SP, Kroenke CH, Laden F, Pukkala E, Schernhammer ES. Night work and breast cancer risk: a systematic review and meta-analysis. Eur J Cancer. 2005;41(13):2023-32.

49. Buja A, Mastrangelo G, Perissinotto E, Grigoletto F, Frigo AC, Rausa G, et al. Cancer incidence among female flight attendants: a meta-analysis of published data. J Womens Health. 2006;15(1):98105.

50. Leggat PA, Smith DR. Dermatitis and aircrew. Contact Dermatitis. 2006;54(1):1-4.

51. Tokumaru O, Haruki K, Bacal K, Katagiri T, Yamamoto T, Sakurai Y. Incidence of cancer among female flight attendants: a meta-analysis. J Travel Med. 2006;13(3):127-32.

52. Michels LR. You're the flight surgeon. Gravity induced loss of consciousness and psoriasis. Aviat Space Environ Med. 2009;80(5):501-2.

53. Griffiths RF, Powell DM. The occupational health and safety of flight attendants. Aviat Space Environ Med. 2012;83(5):514-21.

54. Iglesias R, Gonzalez G, Morales ST. Occupational injuries suffered by flight attendants while on board. Aviat Space Environ Med. 1989;60(11):1109-11.

55. Gil A. Air transport deregulation and its implications for flight attendants. International Labour Review. 1990;129(3):317-31.

56. Linnersjö A, Hammar N, Dammström BG, Johansson M, Eliasch H. Cancer incidence in airline cabin crew: experience from Sweden. Occup Environ Med. 2003;60(11):810-4.

57. Waters MA, Grajewski B, Pinkerton LE, Hein MJ, Zivkovich Z. Development of historical exposure estimates of cosmic radiation and circadian rhythm disruption for cohort studies of Pan Am flight attendants. Am J Ind Med. 2009;52(10):751-61.

58. Banks JO, Avers KE, Nesthus TE, Hauck, EL. A comparative study of international flight attendant fatigue regulations and collective bargaining agreements. JATM. 2012;19:21-4.

59. Pinkerton LE, Yong LC, Yiin JH, Anderson JL, Deddens JA. Cause-specific mortality among a cohort of U.S. flight attendants. Am J Ind Med. 2012;55(1):25-36. 
60. Winkel J. On the manual handling of wide-body carts used by cabin attendants in civil aircraft. Appl Ergon. 1983;14(3):162-8.

61. Murphy AG. Hidden transcripts of flight attendant resistance. MCQ. 1998;11(4):499-535.

62. Brown TP, Shuker LK, Rushton L, Warren F, Stevens J. The possible effects on health, comfort and safety of aircraft cabin environments. J R Soc Health. 2001;121(3):177-84.

63. Burström L, Lindberg L, Lindgren T. Cabin attendants' exposure to vibration and shocks during landing. JSV. 2006;298(3):601-5.

64. Ozcan HK, Nemlioglu S. In-cabin noise levels during commercial aircraft flights. Canadian Acoustics/Acoustique canadienne. 2006;34(4):31-5.

65. Glitsch U. et al. Physical workload of flight attendants when pushing and pulling trolleys aboard aircraft. Int J Ind Ergon. 2007;37(1112):845-54.

66. Jäger M. et al. Load on the lumbar spine of flight attendants during pushing and pulling trolleys aboard aircraft. Int J Ind Ergon. 2007;37(1112):863-76.

67. Lekha S. Lifestyles, flying and associated health problems in flight attendants. J R Soc Health. 2007;127(6):268-75.

68. Mellert V, Baumann N, Freese R, Kruse R, Weber R. Impact of workplace environment on health and comfort of flight attendants and pilots: results from the EU project HEACE. Proceedings of the 25th
International Congress on Aeronautical Science; 2006 Sep 3-8; Hamburg, Germany. 2006. p. 4372-9.

69. Schaub K. et al. Muscular capabilities and workload of flight attendants for pushing and pulling trolleys aboard aircraft. Int J Ind Ergon. 2007;37(11-12): 883-92.

70. Mahony PH. et al. Retention of knowledge and skills in first aid and resuscitation by airline cabin crew. Resuscitation. 2008;76(3):413-8.

71. Mellert V, Baumann I, Freese N, Weber R. Impact of sound and vibration on health, travel comfort and performance of flight attendants and pilots. Aerospace Science and Technology. 2008;12(1):1825 .

72. Agampodi SB, Dharmaratne SD, Agampodi TC. Incidence and predictors of onboard injuries among Sri Lankan flight attendants. BMC Public Health. 2009;11(9):227.

73. Gładyszewska-Fiedoruk K. Indoor air quality in the cabin of an airliner. JATM. 2012;20:28-30.

74. Gupta JK, Lin CH, Chen Q. Risk assessment of airborne infectious diseases in aircraft cabins. Indoor Air. 2012;22(5):388-95.

75. Mallardo V, Aliabadi MH, Brancati A, Marant V. An accelerated BEM for simulation of noise control in the aircraft cabin. Aerospace Science and Technology. 2012;23(1):418-28.

76. Falzon P. Metodologia da ação ergonômica. In: Falzon P, editor. Ergonomia. São Paulo: Edgard Blucher; 2007. p. 3-19. 